\title{
Two dual-wavelength sequences of high-resolution solar photospheric images captured over several hours and restored by use of phase diversity
}

\author{
M. G. Löfdahl ${ }^{1}$, T. E. Berger ${ }^{2}$, and J. H. Seldin ${ }^{3, \star}$ \\ 1 Royal Swedish Academy of Sciences, SCFAB, 10691 Stockholm, Sweden \\ 2 Lockheed Martin Solar and Astrophysics Laboratory, B/252, 3251 Hanover Street, Palo Alto, CA 94304, USA \\ 3 ERIM International, PO Box 134008, Ann Arbor, MI 48113-4008, USA
}

Received 6 June 2001 / Accepted 6 August 2001

\begin{abstract}
The collection, seeing compensation, and temporal filtering of two high-resolution time-sequences of solar photospheric images are described. $44^{\prime \prime} \times 80^{\prime \prime}$ image sequences of co-spatial and co-temporal $430.5 \mathrm{~nm} G$ band and $436.4 \mathrm{~nm}$ continuum filtergrams, were obtained with the $47.5 \mathrm{~cm}$ Swedish Vacuum Solar Telescope. One data set covers 5 hours of photospheric evolution; the other covers 8 hours. The field-of-view contains both an enhanced network region, a large pore, and in the longer sequence, a pore forming event. The mean time between frames is $<40 \mathrm{~s}$. With a few exceptions, the $G$ band frames are partitioned phase-diverse speckle (PPDS) restorations of three realizations of the atmospheric turbulence acquired rapidly in sequence. Due to strict simultaneity and closely spaced wavelengths, the $G$ band wavefronts, compensated for fixed aberration differences, could also be used for deconvolving the corresponding continuum data. For some of the data, collected during periods of particularly bad seeing, restorations made with a related method, joint phase-diverse speckle, were substituted for the PPDS restorations.
\end{abstract}

Key words. methods: observational - techniques: high angular resolution - techniques: image processing Sun: granulation - Sun: magnetic fields - Sun: photosphere

\section{Introduction}

The generation and evolution of small-scale magnetic field structure in the solar photosphere remains unclear. Recently attention has focused on "magnetic elements" the smallest strong-field structures which can be observed interacting with the photospheric flowfield.

The minimum requirements for observation of photospheric magnetic element dynamics are a very high spatial resolution $\left(\lesssim 0^{\prime \prime} 3\right.$; Title \& Berger 1996) with a high degree of uniformity from frame to frame, a large $\left(\sim 1^{\prime}\right)$ field-ofview (FOV), continuous observations at a cadence on the order of $10 \mathrm{~s}$ for periods on the order of hours, and a mode of observation that is sensitive to the magnetic signal. The detailed basis for these requirements is demonstrated by Berger et al. (1998b).

Photospheric bright points (BPs) are seen with particularly high contrast in filtergrams taken in the $430.5 \mathrm{~nm}$ molecular bandhead of CH called the $G$ band (Muller \& Roudier 1984; Berger et al. 1995). Simultaneous images in $G$ band and nearby continuum wavelengths can be subtracted to produce images that trace BPs extremely well

\footnotetext{
Send offprint requests to: M. G. Löfdahl,

e-mail: mats@astro.su.se

* Present address: Essen Instruments, 3909 Research Park Drive, Suite 400, Ann Arbor, MI 48108, USA.
}

(Scharmer 1995). The granulation common to both images is largely eliminated and the difference, composed primarily of magnetic structure, resembles Ca II $K$-line images in that the magnetic elements seem distended, or smeared-out, due to the excess brightness of the $G$ band images near magnetic elements relative to the continuum bandpass (Berger et al. 1998b). Berger \& Title (2001) give further observational evidence that the $G$ band BPs are excellent tracers of photospheric magnetic field concentrations.

Seeing is the primary obstacle to obtaining high resolution time-series observations from the ground. Turbulence in the atmosphere along the line of sight randomly distorts the wavefronts emanating from the Sun. The result is blurring and geometrical distortions in the collected images. Phase Diversity (PD) methods (Gonsalves 1982; Paxman et al. 1992a; Löfdahl \& Scharmer 1994) can estimate the un-aberrated object as well as the aberrations responsible for the blurring. This is accomplished by use of simultaneous images taken in different focus positions, where information about the object and the instantaneous wavefront at the time of exposure are encoded in linearly independent ways. We refer to techniques, where several pairs of PD data with a common object are used to make a single restored frame, as phase-diverse speckle (PDS) 
methods (Paxman et al. 1996). A small number of atmospheric realizations is sufficient for good restorations, significantly reducing the amount of data needed compared to speckle imaging methods. This increases the chances of collecting good data in each sample interval. It also reduces the time needed to write data to disk as well as the total amount of data to be handled for a given sample rate. Another appealing property of phase diversity methods is that the total wavefront of the optical system, including telescopic aberrations, is estimated and corrected for.

A PD data set with the required characteristics was collected and restored from seeing effects by Löfdahl (1996), Löfdahl et al. (1998, henceforth Paper I). The bright point dynamics in the resulting 70-min data sequence was analyzed by Berger et al. (1998a,b), van Ballegooijen et al. (1998), van Ballegooijen \& Nisenson (1999), Krishnakumar \& Venkatakrishnan (1999), Hasan et al. (2000), Lawrence et al. (2001). Also, as demonstrated by Sánchez Almeida et al. (2001), the restored contrasts can be used for comparison with synthesized $G$ band spectra from models of flux tubes.

In this paper we describe the data collection and restoration techniques used to produce $G$ band/continuum data with improvements in optical setup, restoration procedure and, above all, duration. Two time sequences, each consisting of several hundreds of simultaneous $430.5 \mathrm{~nm} G$ band and $436.4 \mathrm{~nm}$ continuum filtergrams, were collected during two consecutive days (see Sect. 2). The $436.4 \mathrm{~nm}$ passband images are termed "continuum" although the bandpass contains several Fraunhofer lines. The FOV includes a plage network region near AR 8050, a large pore or non-penumbral sunspot, and the sequences span periods of $5 \mathrm{~h}$ and $8 \mathrm{~h}$ of photospheric evolution, respectively.

The implementation of partitioned PDS (PPDS) used here has been thoroughly tested with real solar data by Löfdahl \& Scharmer (1994) and Paxman et al. (1996) and we refer to these papers for detailed information about the algorithm. The data processing (see Sects. 3 and 4) is similar to what was used in Paper I, with a couple of notable differences. We use wavefronts estimated in one wavelength channel to restore images from both channels and we use joint PDS (JPDS, Paxman et al. 1992b) in order to obtain better estimates from some data collected during moments of particularly bad seeing.

\section{Observations}

Phase-diverse (PD) data were collected at $430.5 \mathrm{~nm}$ and $436.4 \mathrm{~nm}$ with the $47.5 \mathrm{~cm}$ Swedish Vacuum Solar Telescope (SVST, Scharmer et al. 1985), La Palma, Spain, on 11 and 12 June 1997. The FOV is an area of enhanced network activity (exhibiting many BPs) near the large pore AR 8050 at N28 E25. Five hours of data were collected between 11:11 and 16:16 UT on 11 June and eight hours of data between 09:56 and 18:02 UT on 12 June.

Figure 1 shows the optical arrangement. Two Kodak Megaplus $1.61536 \times 1032$-pixel 10-bit CCD cameras were used. One camera had a center wavelength $430.5 \mathrm{~nm}$

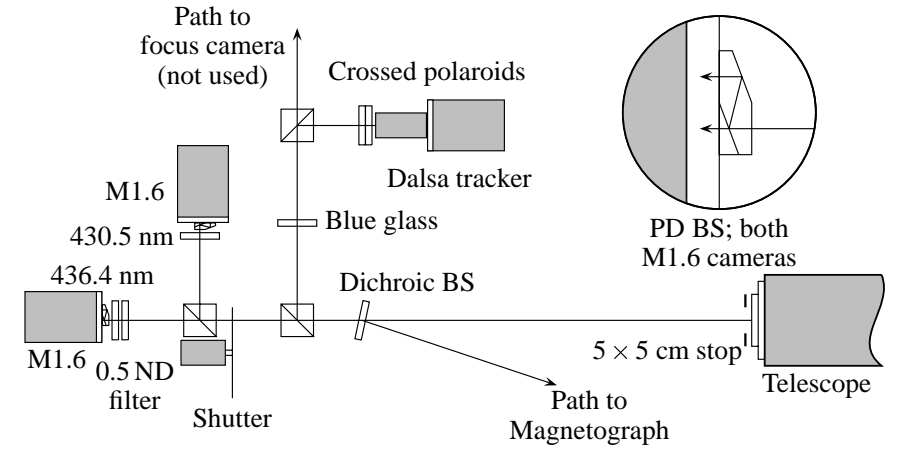

Fig. 1. Optical setup used 11-12 June 1997 at the Swedish Vacuum Solar Telescope, La Palma. The circular inset shows a PD beam-splitter of the kind used in front of the two Megaplus 1.6 cameras.

$G$ band interference filter (1.2 nm bandpass) and the other had a center wavelength $436.4 \mathrm{~nm}$ interference filter (Andover ANDV5405; $1.1 \mathrm{~nm}$ bandpass). The image scale is $0^{\prime \prime} 083$ pixel $^{-1}$; the diffraction limited $(\lambda / D)$ resolution is $00^{\prime \prime} 187$ in the $G$ band and 0.190 in the $436.4 \mathrm{~nm}$ continuum. The integration time was $20 \mathrm{~ms}$.

Both cameras were equipped with PD beam-splitters (see inset in Fig. 1) which put two images on each CCD with a difference in focus position corresponding to $8.85 \mathrm{~mm}( \pm 0.1 \mathrm{~mm})$ in air. This is equivalent to 1.16 and 1.14 waves peak-to-peak of defocus in the $G$ band and in the continuum, respectively.

The optical setup was improved in several ways compared to Paper I. The wavelength difference is smaller, more of the optical path is in common to the two PD cameras and the final beam-splitter is closer to the focus plane. The external shutter, common to the two cameras, gave strictly simultaneous exposures between cameras and between corresponding parts of the FOV in the PD channels. These improvements reduced both the systematic and the non-systematic wavefront differences between the cameras. The motion of the external shutter was perpendicular to the diversity channel divider, which obviated some simultaneity problems between diversity channels experienced with the previous setup.

Telescope pointing and jitter correction were accomplished in real-time using a spot tracking system. This system uses a Dalsa CA-D1-0128 CCD camera with a blue filter to acquire images of the spot. The images are read at 820 frames per second through a DMA interface to a DEC Alpha workstation, to be interpreted as quad cell data. Offsets calculated to balance the spot area between quarter planes of the CCD are translated to commands that are sent to the servo-control of the M3 mirror of the SVST. The spot tracking system gives stable subarcsecond pointing during virtually any seeing conditions.

Frame selection was carried out on the $G$ band camera; the best three frames from each $30 \mathrm{~s}$ selection period, and the simultaneous continuum frames, were recorded for later processing. Intervals of $30 \mathrm{~s}$ are used in order to sample over a wide range of seeing conditions while also ensuring that minimal solar evolution occurs between the 
selected images from adjacent intervals. With overhead for writing the images to disk, the average cadence of the selection intervals is $36 \mathrm{~s}$. The frames that are selected are usually recorded within seconds of each other since good seeing tends to occur intermittently. This justifies the assumption of a common object for the three image pairs. Dark frames were collected at the end of the day by blocking the beam from the telescope. Flat fields were collected, also at the end of the day, by moving the table $20 \mathrm{~cm}$ out of focus and rapidly scanning an area of quiet sun by oscillating the M3 mirror. All images were dark-frame subtracted and flat-field corrected. Local median values were substituted for the spikes associated with a small number of hot pixels in the continuum camera.

\section{Wavefront sensing and image restoration}

\subsection{June 1997, 5-hour data set}

\subsection{1. $G$ band $430.5 \mathrm{~nm}$ preprocessing}

The diversity channels were brought into approximate registration by cross-correlating a small number of focused images with their corresponding defocused images.

A subset of the $G$ band data was selected for preprocessing in order to get good estimates of the sub-pixel registration parameters between the diversity channels, while at the same time monitoring their evolution in time. We chose the locally best image pair (based on the frame selection sharpness value) from every group of roughly 25 consecutive frames. These data were pre-processed with the PD program, estimating sub-pixel inter-channel registration. Using only good quality data for this step lets the poorer data benefit from the parameters estimated from the better data. The registration parameters, averaged over the subfields and linearly fitted to the observation time, were then kept fixed during the main inversions described in Sect. 3.1.2. In order to avoid misinterpretation of coma-type aberrations as registration parameters, a high-order expansion of the wavefronts (Zernike polynomials $Z_{4}$ through $Z_{45}$ as defined by Noll 1976) was used in this step. The noise variance ratio between diversity channels was measured and averaged to 0.444 .

\subsection{2. $G$ band PPDS processing}

Images are restored by deconvolution using a low-pass filter, rejecting the high frequencies that are dominated by noise. From any pair of PD images, information can also be missing at isolated lower frequencies, where the modulation transfer function happens to be zero in both PD channels. This can cause ringing in the restored images, even when the estimated wavefronts are correct. The location of these isolated frequencies vary with time, so information can be filled in by basing the restorations on several image pairs, and the corresponding wavefront estimates, as described by Löfdahl \& Scharmer (1994), Paxman et al. (1996). We call this technique Partitioned Phase Diverse Speckle (PPDS) imaging because a PDS data set with several exposures close in time is used, although the wavefront sensing is partitioned. We refer to Paper I for the details of PPDS processing of $G$ band data. Here we give enough detail to point out differences from the processing of that set.

Except for the subfield size, the wavefronts were estimated using the same parameter settings as used in Paper I: The highest Zernike mode used was $Z_{21}$ and the SVD cutoff parameter was set to $w_{\mathrm{c}}=0.08$. Apodization of subfields was done with a modified Hanning window.

The error metric and its derivatives were evaluated over $80 \times 80$-pixel $\left(6^{\prime \prime} .6 \times 66^{\prime \prime} 6\right)$ subfields, while the FFTs were calculated on $128 \times 128$-pixel subfields. This gave an effective 24 pixels wide guard band from FFT wraparound errors. Aside from the guard band, the size of the error metric evaluation window is also a tradeoff, in that large subfields contain more information that can facilitate the inversions but small subfields avoid averaging out anisoplanatic wavefront variations. Previous experience with data from the SVST indicates an isoplanatic angle of $\sim 5^{\prime \prime}$ as a reasonable approximation (Löfdahl \& Scharmer 1994; Paper I). Visual inspection (blind testing) of difference images from a small set of varying quality data indicated that $80 \times 80$ pixels was a better tradeoff for this data set, perhaps due to less anisoplanatism. This increased the area per subfield by $30 \%$ and thereby reduced the processing time by the same amount.

The full $G$ band data set consists of 1540 phase-diverse image pairs. With $84(7 \times 12)$ subfields per frame, the total number of wavefront determinations is $1.3 \times 10^{5}$. The average number of iterations required to converge wavefront corrections to $<0.01 \mathrm{rad} \mathrm{rms}$ was 6.0 . The restored subfields are mosaicked so that reconstructions of the entire $466^{\prime \prime} 5 \times 79^{\prime \prime} .7(560 \times 960$-pixel $)$ FOV are obtained.

\subsubsection{Deconvolution of continuum $436.4 \mathrm{~nm}$ data by use of $G$ band wavefronts}

All the continuum data were restored using wavefronts calculated from the corresponding $G$ band wavefront estimates, reducing the number of inversions by a factor of two (not counting the small number of inversions needed to find the systematic aberration differences). Because of synchronization errors on the order of $8 \mathrm{~ms}$, this procedure was not possible with the inferior optical setup described in Paper I. The linear relations that were used to convert from $G$ band to continuum Zernike coefficients, taking wavelengths and systematic aberration differences into account, are fits to corresponding wavefront estimates from both wavelength channels. The selected subset of good quality frames used in Sect. 3.1.1 was used for estimating sub-pixel inter-channel registration parameters. Then that set as well as the varying-quality set from Sect. 3.1.2 were used for estimating wavefronts with fixed registration parameters. As can be seen in Fig. 2, there is excellent consistency between the wavefronts estimated in the two wavelengths. The correlation coefficients are $>0.8$ for all modes and $>0.9$ for most. Without the varying-quality set the correlation is not as high, due to a smaller range of measured values. 


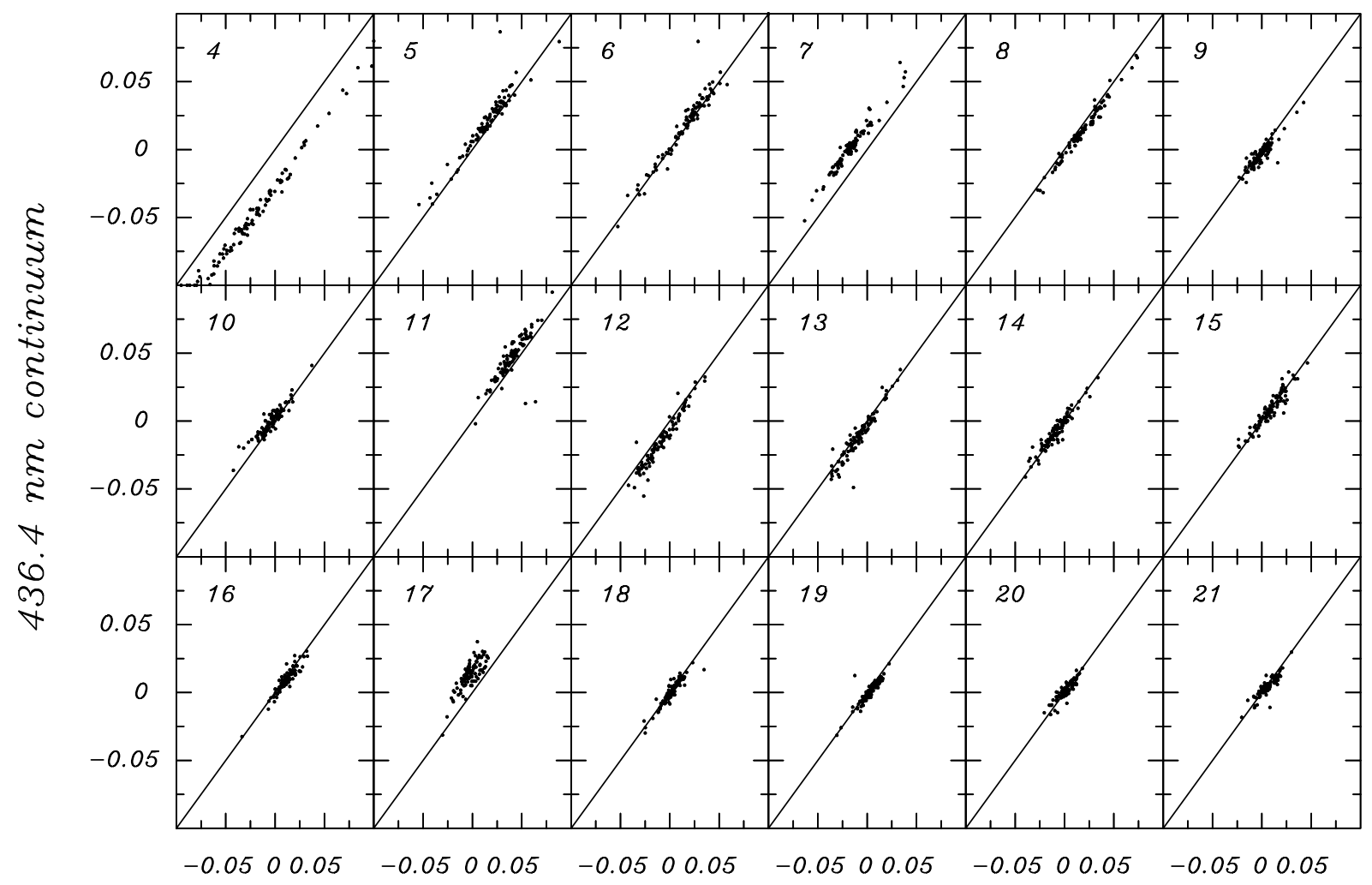

$430.5 \mathrm{~nm} \mathrm{G}$ band

Fig. 2. Scatter plots of continuum vs. $G$ band subfield-averaged Zernike coefficients in waves from a selected subset of good quality frames, $\left\langle\alpha_{m}^{436.4}\right\rangle$ vs. $\left\langle\alpha_{m}^{430.5}\right\rangle$. The Zernike indices, $m$, are shown in the upper left corner of each tile. The low-order indices correspond to focus $(m=4)$, astigmatism $(5,6)$, coma, $(7,8)$, triangular coma $(9,10)$, and spherical aberration (11). A few points are outside the plotting area in the focus tile.

Continuum images from another subset of data with varying quality, restored using converted $G$ band wavefronts, look better (according to a blind testing experiment) than images restored using wavefronts calculated directly from the continuum data. This can be explained by a combination of different effects. As noted in Paper I, the $G$ band images contain more high-frequency information, which may allow higher wavefront modes to be estimated correctly. Also, when there are wavefront differences between the two cameras, the frame selection procedure gives a bias to smaller wavefronts in the selection camera - the $G$ band in this case. Smaller wavefronts give better wavefront estimates and the average wavefront rms in the two channels, $39 \mathrm{~nm}$ in the $G$ band and $45 \mathrm{~nm}$ in the continuum channel $(34 \mathrm{~nm}$ and $37 \mathrm{~nm}$, resp., excluding focus), is consistent with such a selection effect.

The two cameras were not perfectly aligned. This was not fully taken into account when choosing the subfield positions for the $G$ band PD processing. One row of $G$ band subfields therefore does not quite fit within the FOV of the continuum camera. In order to maximize the common FOV, the corresponding continuum subfield positions were shifted and $G$ band wavefronts calculated by linear interpolation between neighboring subfields were used. The interpolation resulted in no apparent loss of image quality. The final size of the continuum restorations and also of the common FOV between the two wavelength channels, is $43^{\prime \prime} .6 \times 799^{\prime \prime} 7=525 \times 960$ pixels.

Figure 2 can also be used to demonstrate the effects of the improvements in the optical setup. Comparison with the corresponding results from the older data set (Paper I; Fig. 3) indicates that the improvements in the optical setup were effective. Whereas the latter figure shows significant systematic differences in a majority of the modes, Fig. 2 indicates that such differences are restricted to modes 4,7 , and 17 . The BS is much closer to the focal planes in the present setup, which is consistent with smaller astigmatism $\left(Z_{5}\right.$ and $\left.Z_{6}\right)$ and spherical aberration $\left(Z_{11}\right)$ differences. The smaller wavefront differences are also consistent with reduced differences in the amounts of glass and optical surfaces between the optical paths to the two cameras. We conclude that the cube BS should be as close to the cameras as physically possible. Differences in focus $\left(Z_{4}\right)$ are of course almost inevitable with the use of two separate cameras and from experience we know that there is often cross-talk in the estimation of interchannel registration parameters and modes that have a tilt component, such as coma and higher order coma. It is somewhat surprising that the differences in $Z_{7}$ and $Z_{17}$ are not repeated in the corresponding modes with perpendicular tilt components, $Z_{8}$ and $Z_{16}$. We note however that in the registration parameter estimation step, there was a 
corresponding symmetry break in that one of the registration parameters was almost constant in time, while the other changed significantly over the course of the observations. We don't know why this happens but note that the effects seen in Fig. 2 are small.

\subsubsection{Fixes for poor restorations}

For some cases, when the restored object quality was below average, particularly as seen in the difference images, attempts were made to subjectively improve the quality.

Including only one or two of the three data frames in a selection interval sometimes helps when the assumption of a common object is violated because the data were collected too far apart in time or when only one or two decent quality frames were collected. In the former case the restored image is blurred because it is a kind of average of the object in the different frames. In the latter case, the PD wavefront sensing failed due to the high aberration content, which in turn led to poor restoration quality. A few frames were restored with less than three frames because of magnetic tape failures.

The restorations can sometimes be improved by slightly decreasing the passband of the spatial filter used in the restoration process $(H$, see Paper I). This can be done simply by adopting a noise rms that is larger than the measured value by a factor 2 .

\subsubsection{JPDS for even poorer datasets}

PPDS reconstructions from some selection intervals were particularly bad. An alternative to the partitioned approach is to jointly estimate wavefronts from, and restore the full PDS data set from, a selection interval. We refer to this technique as Joint Phase Diverse Speckle (JPDS, Paxman et al. 1992b). Better results are expected with JPDS because the joint treatment constrains the wavefronts by requiring that the object be identical in all frames. Because of this, the parameters are better determined than in the PPDS case. In addition to just giving better accuracy, this advantage can be utilized in different ways. In bad seeing, high-order aberrations are more likely to affect the data and JPDS allows more parameters to be determined. Smaller subfields can be used, which should help when there is strong anisoplanatism.

60 selection intervals, including those with the very worst PPDS restorations, were also processed with JPDS. We refer to Seldin \& Paxman (1994), Paxman et al. (1996) for prior work on solar image restoration with JPDS. The following details describe the processing of the current data. FFTs were performed on $96 \times 96$-pixel arrays and the error metric was evaluated over $80 \times 80$ pixels. This gives an 8-pixel guard band for wrap-around errors. The restorations are mosaics on a 72-pixel grid. The wavefronts were parameterized using Zernike polynomials 2-66, thus using the JPDS advantage to estimate a larger number of parameters. A Gaussian sieve with $\sigma=0.5$ pixels was used.

From the set processed with JPDS, 39 restorations (from each wavelength channel) were selected for inclusion in the final set of restored data because they are significantly better than the corresponding PPDS restorations. In at least four cases this filled in gaps of several selection intervals where the PPDS restorations would not deliver restorations that were good enough to be included in the set at all.

\subsubsection{The restored data set}

The overall quality of the restored data is comparable to the data presented in Paper I. Figure 3 shows a sample restored $G$ band image, demonstrating the $43^{\prime \prime} .6 \times 79^{\prime \prime} 7$ $(525 \times 960$-pixel $)$ FOV that is in common to the two wavelength channels. A total of 516 restored images were included in the final set. 437 frames were PPDS restored using three PD pairs, 36 using two pairs and 4 using single pairs. Noise artifacts were fixed in 11 PPDS frames by manipulating the filter 39 frames in the sequence were restored with JPDS.

The 11 June dataset has a roughly normal distribution of time between frames with a minimum of $14 \mathrm{~s}$, a maximum of $136 \mathrm{~s}$, a mean of $36 \mathrm{~s}$, and a standard deviation of $11 \mathrm{~s}$. The largest gaps (on the order of $\$ 2 \mathrm{~min}$ ) in the data sequence are due to data frames that could not be read off one of the tapes.

Figure 4 shows a comparison of raw focused data and PPDS restorations for a set of good data. Note how also the bright granules cancel in the associated difference images. The PPDS images are noticeably better resolved, particularly in the difference image.

Although the brightness and contrast are expected to be different in the two wavelengths, the morphology should be similar. We use this to demonstrate qualitatively how sample JPDS results are superior to the corresponding PPDS results. In Fig. 5 it is clear that the PPDS restorations have less contrast than the JPDS restorations, particularly in the continuum. The PPDS continuum restoration is so blurry, that the difference image looks like a bad copy of the $G$ band image. The JPDS reconstructions have much more uniform quality and the resolution is better, although missing high spatial frequency information is apparent also in the JPDS restorations.

For a more subtle example, the restorations in Fig. 6 appear to be about the same quality. The granulation is largely canceled out in both difference images. However, closer examination of the PPDS restorations show many fine structures that are morphologically different in the two wavelengths, where the corresponding JPDS restorations are more similar. These structures are easily spotted by inspecting the filtergrams at locations corresponding to black patches in the PPDS difference image. The JPDS difference image has much less background structure than the PPDS difference image, and primarily in the form of evenly distributed "mottle". The PPDS difference image has a lot of fringe-like artifacts, indicating that it would only be worse if more high frequency information were included. 


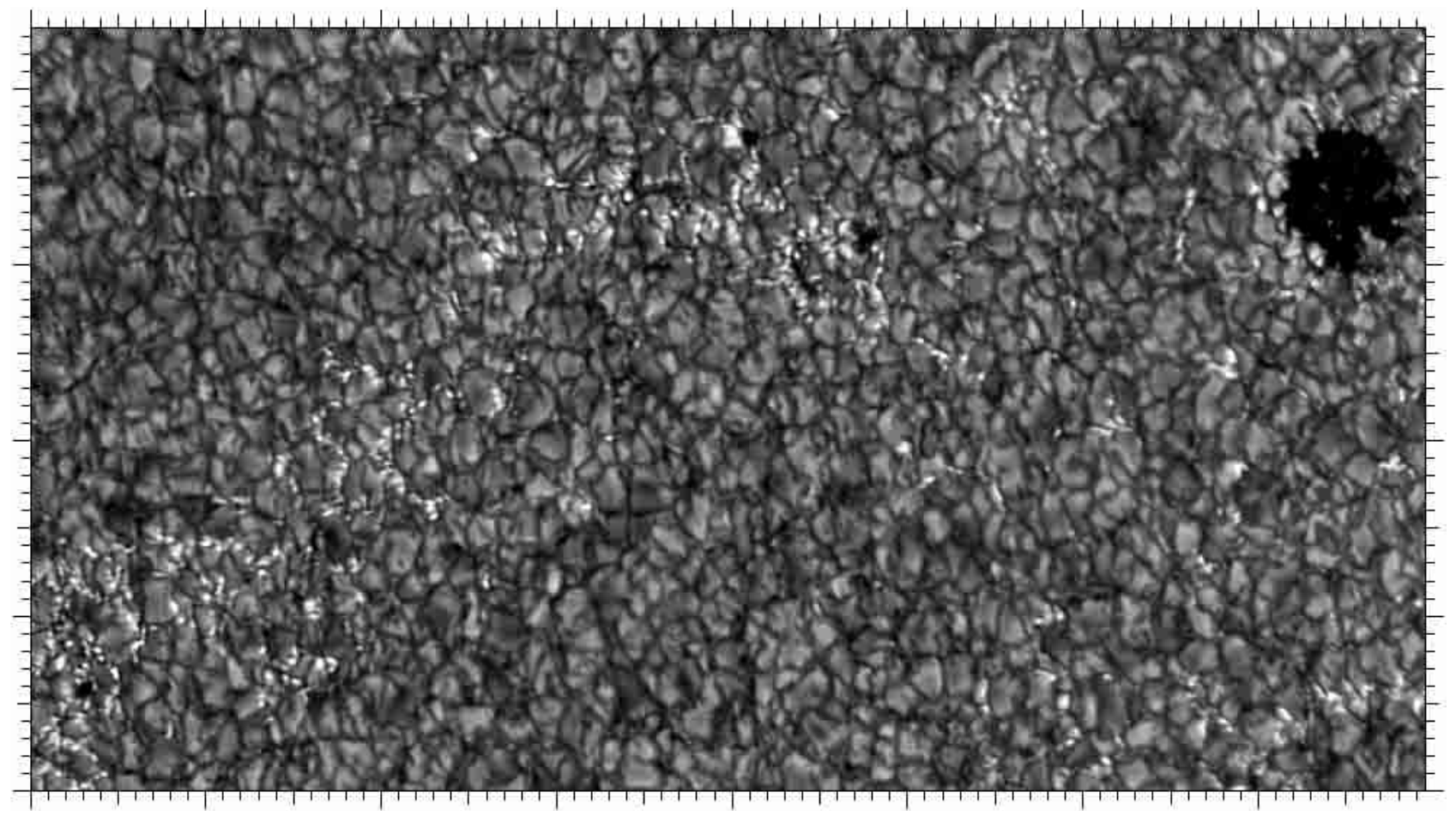

Fig. 3. $430.5 \mathrm{~nm} G$ band filtergram: 1997 June 11, 14:31 UT (frame 342) plage network region near the pore AR 8050 at N28 E25; Kodak Megaplus 1.6 camera; exposure $20 \mathrm{~ms}$; plate scale $=12$ pixels $\operatorname{arcsec}^{-1}$. Tic marks are $1^{\prime \prime}$ apart.

Turning attention back to PPDS and some of the better data frames, we note in Fig. 7 that the restored images show a lot of well resolved umbral dots in the pore. However, this is also where we can see difference image artifacts also for the very good frames. For several quite good restored frames, the umbral dots show up in the difference images with reversed contrast with respect to the $G$ band bright points. The reversed contrast does not appear, however, in the very best frames, so we conjecture that it is an artifact from the reconstruction process.

\subsection{June 1997, 8-hour data set}

The FOV is similar to Fig. 3, but the pore is along one of the long edges, about one third of the distance from one of the corners. It is also not completely within the common FOV of the two wavelength channels.

These data were processed similarly to the processing of data from 11 June, as described in Sects. 3.1.1 through 3.1.4. However, JPDS was not used. The average number of $G$ band PD iterations was 6.5. Approximately 4 frames per 100 ( 2 selected as locally best and 2 as average quality) were used for establishing the $G$ band-continuum wavefront relations. The correlations between $G$ band and continuum wavefronts are similar to the data from the previous day, see Fig. 2, including the high correlation coefficients and the offsets for $Z_{7}$ and $Z_{17}$.

The restored FOV is $8 \times 12$ subfields in $G$ band and $7 \times 12$ in continuum. To fit in the raw data frame, the continuum subfields were displaced by 8 pixels with no apparent problem. There was no extra strip added to the continuum FOV.
709 frames were restored using three PD pairs, 81 using two pairs, and 9 using single pairs, giving a total of 799 restored frames. Artifacts were fixed with the filter in 4 of them. The 12 June dataset has a distribution of time between frames that is similar to the 11 June data, except for a couple of longer gaps (the longest at $460 \mathrm{~s}$ ) due to occasional synchronization failure between the $G$ band and continuum cameras resulting in a restart of both control programs.

\section{Geometrical corrections}

The restored and mosaicked images were further processed to remove geometric mismatches. The images were rotated to remove the field rotation from the alt-az mount of the telescope. The rotation amounted to a total of $58^{\circ}$ from end point to end point over the course of the 5 -hour set from 11 June. The corresponding angle for the 8-hour 12 June data set is $95^{\circ}$. Because the spot tracker was used during the observations, the center of rotation is the pore. The position of the pore in the FOV made the FOV better preserved in the 12 June data than in the data from the previous day. This step also included a registration step, that removed residual global tilts.

Anisoplanatism gives geometric distortion between frames. The relative distortions were removed by "temporal destretching" as described by Shine et al. (1994) (see also Topka et al. 1986; Tarbell et al. 1979). This method assumes that high frequency distortions are atmospheric in origin and attenuates them. Lower frequency motions due to real surface flows are preserved. However, any high frequency solar motions are attenuated. 

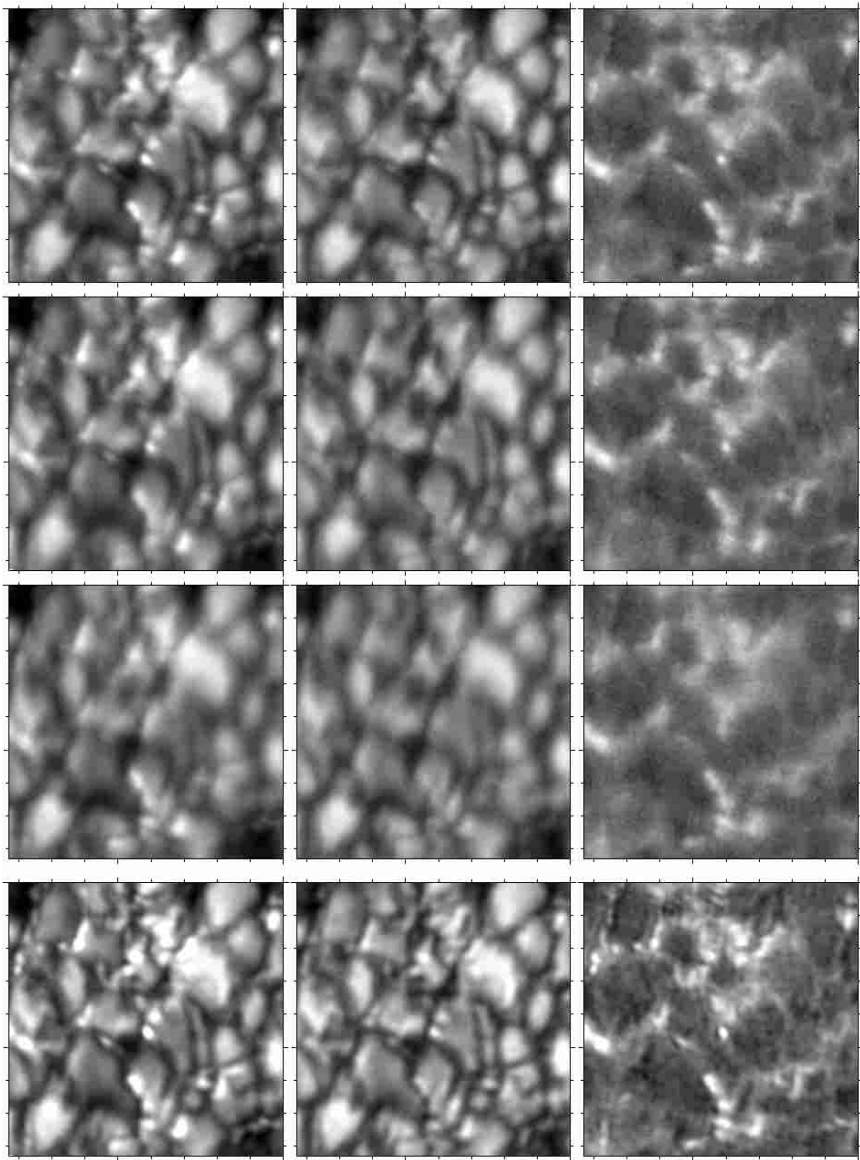

Fig. 4. Example of good data. Top three rows: raw focused data; bottom row: PPDS restoration. Left: $G$ band; center: continuum; right: $G$ band-continuum difference. Tic marks are $1^{\prime \prime}$ apart.
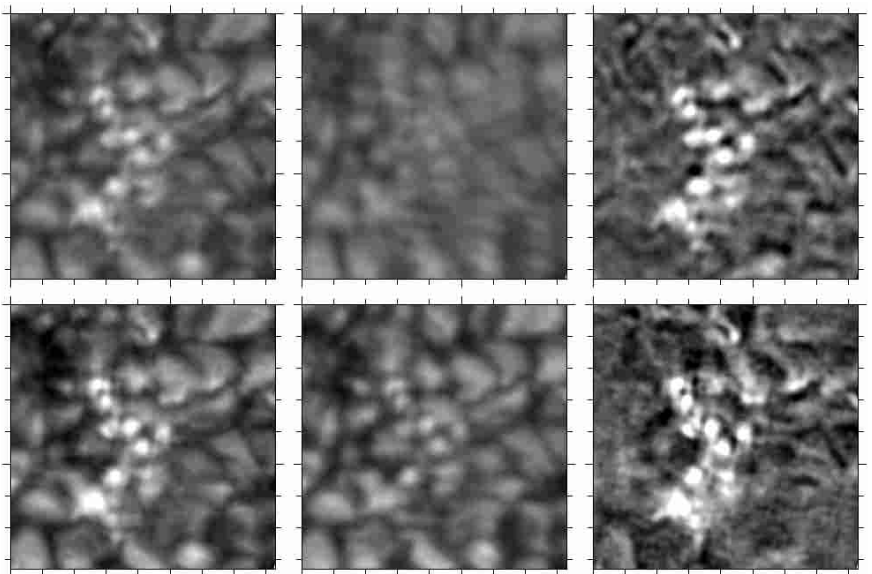

Fig. 5. Comparison of PPDS restoration and JPDS restoration: large-scale effects. Top: PPDS; bottom: JPDS. Left: $G$ band. Center: continuum. Right: difference. Tic marks are $1^{\prime \prime}$ apart.

\section{Discussion}

As demonstrated in Paper I, PDS image restoration of solar photospheric data can produce the high quality sequences of data necessary for measuring subgranular scale flowfields in the photosphere. We expect the dataset presented here, in addition to $G$ band BP work already in
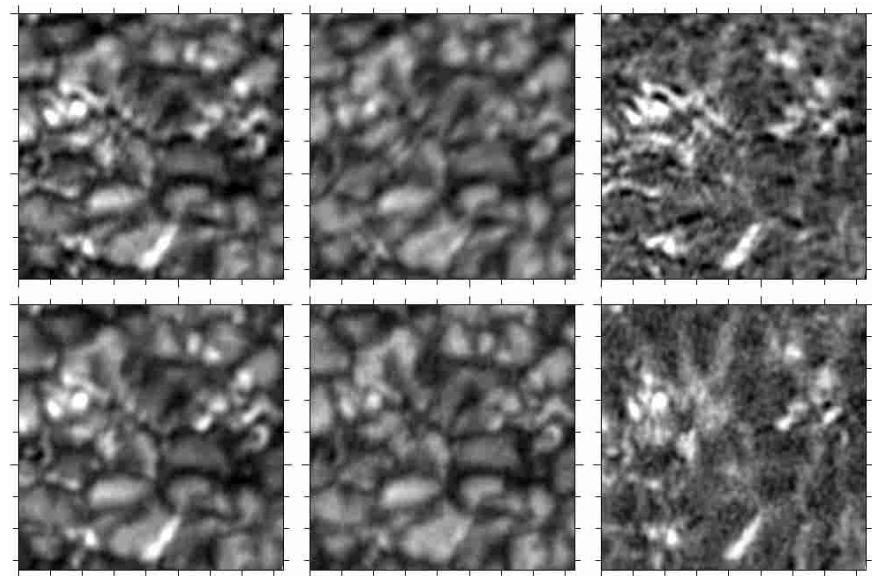

Fig. 6. Comparison of PPDS restoration and JPDS restoration: small-scale effects. Top: PPDS; Bottom: JPDS. Left: $G$ band. Center: continuum. Right: difference. Tic marks are $1^{\prime \prime}$ apart.
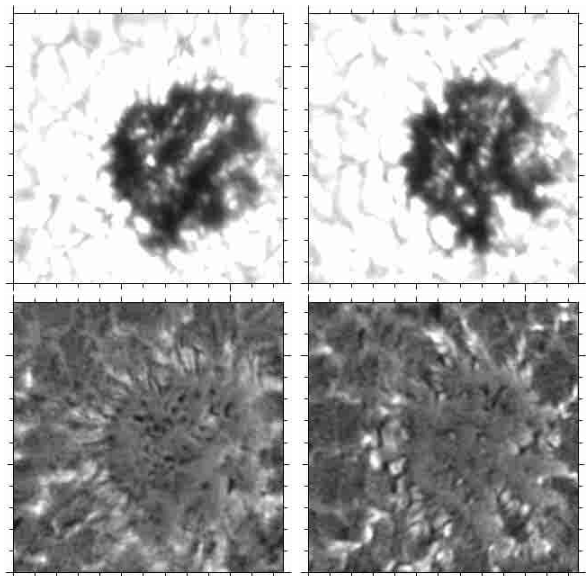

Fig. 7. Two different $G$ band (top) and difference (bottom) images of the pore. Left: good restoration with reversed contrast umbral dots in the difference image; Right: better restoration with significantly less reverse contrast umbral dots. Tic marks are $1^{\prime \prime}$ apart.

progress, to also be useful for local helioseismology, convection studies, etc. Some of the 11 June data has already been used by Kiselman et al. (2001) for comparison with synthesized spectra from models of flux tubes.

We would like to emphasize that the improvements in the optical setup from the earlier campaign (Paper I) were necessary for the time-saving and quality enhancing technique of restoring both wavelength channels using wavefronts from the channel used for frame selection. In addition to the subjective impressions when comparing continuum PPDS restorations and continuum frames restored with $G$ band wavefronts, rigid alignment (rotation and shift) was enough to bring $G$ band and continuum into alignment this time. This is in contrast to Paper I, where stretching was necessary to co-align the images properly, indicating anisoplanatism evolution between the exposures in the two wavelength channels. We attribute this improvement to the common external 
shutter, which ensures strictly simultaneous image recording in the two cameras.

Based on our favorable experience with PPDS from a previous data set (Paper I), we used PPDS to restore most of the data reported here, and it was indeed adequate for most of the frames. However, although Paxman et al. (1996) found no significant improvement in restored image quality with JPDS over PPDS using data collected under good seeing conditions, the worst of the current data is clearly a regime where JPDS has advantages. When tried on some of the harder cases, JPDS gave (sometimes dramatically) superior restorations which were therefore substituted for the PPDS restorations, although there were also cases when PPDS restorations were marginally better. Since strong geometrical distortions from anisoplanatism would affect JPDS already in the wavefront sensing step by violating the assumption of a common object, we examined statistics of relative stretching on sizes smaller than the $80 \times 80$-pixel patches used for restoration and mosaicking. We found no excess of PPDS superiority at large stretching values. We conclude that JPDS is often better and that further study is needed to find the optimal PDS processing strategy, particularly since there are other differences than the joint/partitioned processing (such as noise model, regularization, optimization algorithms, etc.) between the two methods used in this paper. We found no reason to rerun the already successful PPDS restorations. Although the comparison of PPDS with JPDS is not the focus of this paper, we have nevertheless expanded somewhat on the findings by Paxman et al. (1996) by demonstrating that for solar data, there is a regime where JPDS performs better than PPDS.

Evaluating the quality of the restored frames is necessary for identifying cases that need more work and for choosing between alternative restorations. Doing this manually is a tedious process for data sets as large as the ones presented here. For automatic evaluation, a metric would be needed that take restored resolution as well as artifacts into account. Formulating such a metric would be a very important progress for large dataset PD restoration.

Acknowledgements. M. G. L. and T. E. B. were supported by NASA SR\&T contract NASW-98008 and Lockheed Martin Space Systems/LMSAL Independent Research Funds. In addition, M. G. L. was supported by the MDI project at Stanford and LMSAL, NASA grant NAG5-3077. J. H. S. was supported by the National Science Foundation Advanced Technologies and Instrumentation Program, NSF Grant No. AST-9817796. Darrel Torgerson performed the optical layout at the SVST. We are grateful to Göran Scharmer for comments and encouragement. The SVST is operated by the Royal Swedish Academy of Sciences in the Spanish Observatorio del Roque de los Muchachos of the Instituto de Astrofísica de Canarias (IAC).

\section{References}

Berger, T. E., Löfdahl, M. G., Shine, R. A., \& Title, A. M. 1998a, ApJ, 506, 439

Berger, T. E., Löfdahl, M. G., Shine, R. A., \& Title, A. M. 1998b, ApJ, 495, 973

Berger, T. E., Schrijver, C. J., Shine, R. A., et al. 1995, ApJ, 454,531

Berger, T. E., \& Title, A. M. 2001, ApJ, 553, 449

Gonsalves, R. A. 1982, Opt. Eng., 21, 829

Hasan, S. S., Kalkofen, W., \& van Ballegooijen, A. A. 2000, ApJ, 535, L67

Kiselman, D., Rutten, R. J., \& Plez, B. 2001, in Recent Insights into the Physics of the Sun and Heliosphere - Highlights from SOHO and other Space Missions, ed. P. Brekke, B. Fleck, \& J. B. Gurman, ASP Conf. Ser., IAU Symp. 203 , in press

Krishnakumar, V., \& Venkatakrishnan, P. 1999, Sol. Phys., 186, 43

Lawrence, J. K., Cadavid, A. C., Ruzmaikin, A., \& Berger, T. E. 2001, Phys. Rev. Lett., in press

Löfdahl, M. G. 1996, Ph.D. Thesis, Stockholm University

Löfdahl, M. G., Berger, T. E., Shine, R. A., \& Title, A. M. 1998, ApJ, 495, 965 (Paper I)

Löfdahl, M. G., \& Scharmer, G. B. 1994, A\&AS, 107, 243

Muller, R., \& Roudier, T. 1984, Sol. Phys., 94, 33

Noll, R. J. 1976, J. Opt. Soc. Am., 66, 207

Paxman, R. G., Schulz, T. J., \& Fienup, J. R. 1992a, J. Opt. Soc. Am. A, 9, 1072

Paxman, R. G., Schulz, T. J., \& Fienup, J. R. 1992b, in Technical Digest Series, vol. 11, Signal Recovery and Synthesis IV, Optical Society of America, 5

Paxman, R. G., Seldin, J. H., Löfdahl, M. G., Scharmer, G. B., \& Keller, C. U. 1996, ApJ, 466, 1087

Sánchez Almeida, J., Asension Ramos, A., Trujillo Bueno, J., \& Cernicharo, J. 2001, ApJ, 555, 978

Scharmer, G. B. 1995, private communication

Scharmer, G. B., Brown, D. S., Pettersson, L., \& Rehn, J. 1985, Appl. Opt., 24, 2558

Seldin, J. H. \& Paxman, R. G. 1994, in Proc. SPIE, vol. 2302, Image reconstruction and restoration, ed. T. J. Schultz, \& D. L. Snyder

Shine, R. A., Title, A. M., Tarbell, T. D., et al. 1994, ApJ, 430,413

Tarbell, T. D., Title, A. M., \& Schoolman, S. A. 1979, ApJ, 229,387

Title, A. M. \& Berger, T. E. 1996, ApJ, 463, 797

Topka, K. P., Tarbell, T. D., \& Title, A. M. 1986, ApJ, 306, 304

van Ballegooijen, A. A., \& Nisenson, P. 1999, in High Resolution Solar Physics: Theory, Observations and Techniques, ed. T. Rimmele, R. R. Radick, \& K. S. Balasubramaniam, Proc. 19th Sacramento Peak Summer Workshop, ASP Conf. Ser., 183, 30

van Ballegooijen, A. A., Nisenson, P., Noyes, R. W., et al. 1998, ApJ, 509, 435 